\title{
Integrated spectroscopy and individual spectra of stars of open cluster remnants and candidates
}

\author{
D.B. Pavani ${ }^{1}$, E. Bica ${ }^{2}$, A.V. Ahumada ${ }^{3}$ \\ and J.J. Clariá ${ }^{3}$ \\ ${ }^{1}$ IAG/USP - Rua do Matão 1226; 05508-900, São Paulo, SP; Brazil \\ email: daniela@astro.iag.usp.br \\ ${ }^{2}$ UFRGS/IF - CP 15051, 912501-970, Porto Alegre, RS; Brazil \\ ${ }^{3} \mathrm{OAC}$ - Laprida, 854, 5000 Córdoba, Argentina \\ email: andrea@mail.oac.uncor.edu
}

\begin{abstract}
The integrated light of open cluster remnants (OCRs) is expected to be similar to that of open clusters of comparable ages. Despite erosion of the mass function in remnants as a consequence of dynamical evolution, their integrated spectra could still be similar to those of the clusters of comparable age as long as a sufficient number of bright stars remain. In the present work we employ integrated spectroscopy and individual spectra of stars to analyze 8 OCRs and candidates which were previously classified as open clusters and a new unclassified object. The OCRs and possible open cluster remnants (POCRs) are underpopulated with respect to usual open clusters, but they show a significant density contrast of bright stars when compared to the Galactic field. The spectroscopic observations were carried out with the $2.15-\mathrm{m}$ telescope at CASLEO (San Juan, Argentina) in the range 3800-6800 A, during 2003 and 2004. We derive foreground interstellar reddening values and ages. We find in most of the sample that the derived properties are compatible with what should be expected for OCRs.
\end{abstract}

Keywords. open clusters and stellar associations, open cluster remnants, spectroscopy.

OCRs correspond to the final evolutionary stages of the open star clusters. The present work is part of a more extensive study that intends to characterize OCRs (Pavani \& Bica 2007). In general terms, ages, reddening values and distances of OCRs are obtained from colour-magnitude diagrams and/or from single star photometric studies. However integrated spectroscopy and individual spectra of stars are useful complementary methods of the photometry. In this work the integrated spectra were obtained setting the slit in the east-west direction and scanning the object in the north-south direction in order to sample the stellar content adequately. We used a CCD camera with $1024 \times 1024$ pixels attached to the REOSC spectrograph. One pixel corresponds to $0.94^{\prime \prime}$ on the sky and the long slit corresponds to $4.7^{\prime}$. The slit width was $4.2^{\prime \prime}$ (resolution $\sim 17 \AA$ ). For reductions we used the IRAF package. The spectra obtained in different runs were averaged by weighting them according to the signal-to-noise ratio. For the spectra containing all probable stars of POCRs we compared their Balmer equivalent widths with those of star cluster templates from Piatti et al. (2002). We fitted the integrated spectra to the closest match among templates to obtain ages and the foreground-reddening values. We repeated the procedure for the spectra dominated by a few bright stars and for individual stars. Finally, we compared the Balmer lines with those of the templates of the stellar library by Silva \& Cornell (1992). The relation between spectral types and ages was obtained from Mermilliod (1981) and/or from Tables 3.7 and 3.10 from Binney \& Merrifield (1998), using solar metallicity Padova isochrones (Girardi et al. 

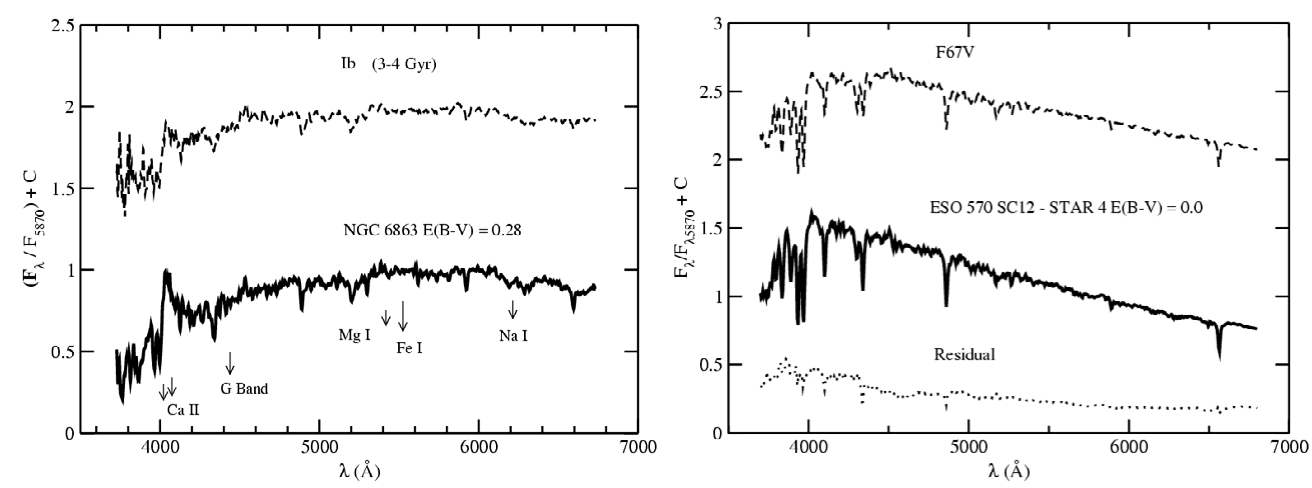

Figure 1. Left panel: example of an observed integrated spectrum corrected for the derived foreground-reddening value compared with a star cluster template from Piatti et al. (2002). Bands and lines of POCRs are shown. Right Panel: spectrum of an individual star compared with a stellar template from Silva \& Cornell (1992). The residual flux of the closest match is shown.

Table 1. Parameters of OCRs and POCRs

\begin{tabular}{lrrcr}
\hline Name & $l$ & $b$ & $\mathrm{E}(\mathrm{B}-\mathrm{V})$ & Age \\
\hline NGC 648 (OCR) & 29.94 & 14.93 & 0.12 & $3-4 \mathrm{Gyr}$ \\
NGC 6863 (OCR) & 38.27 & -14.99 & 0.28 & $3-4 \mathrm{Gyr}$ \\
RUPRECHT 31 (POCR) & 250.08 & -5.97 & 0.35 & $<0.1 \mathrm{Gyr}$ \\
ESO 211 SC8 (POCR) & 271.51 & -0.43 & 1.30 & $<0.2 \mathrm{Gyr}$ \\
OBJECT 1 (POCR) & 294.94 & -0.63 & 0.01 & $<1.0 \mathrm{Gyr}$ \\
ESO 132 SC14 (POCR) & 308.10 & 0.20 & 0.35 & $<1.0 \mathrm{Gyr}$ \\
ESO 383 SC10 (OCR) & 312.13 & 27.08 & 0.00 & $<2.0 \mathrm{Gyr}$ \\
ESO 389**5 (POCR) & 339.91 & 12.94 & 0.00 & $<2.0 \mathrm{Gyr}$ \\
ESO 570 SC12 (OCR) & 274.06 & 35.89 & - & - \\
\hline
\end{tabular}

2002). Examples of the spectra and of the closest matches are shown in Fig. 1. For two stars of ESO 570 SC12 we obtained spectral types F8 and F6, respectively. Table 1 shows the ages and the reddening values inferred for the present sample.

We present for the first time ages, reddening values and spectral types for a sample of OCRs and POCRs obtained from integrated spectroscopy and/or from individual spectra of stars. These preliminary results will be combined with parameters previously determined from 2MASS photometry for the best characterization of the objects. The integrated spectra, specially those containing all probable members of POCR provide observational evidence of the existence of OCRs.

\section{Acknowledgements}

We would like to thank the Brazilian institutions FAPESP and CNPq for their financial support. We are also indebted to the CASLEO staff members for their help during the observing runs.

\section{References}

Binney, J-C. \& Merrifield, M. 1998 in: Galactic Astronomy, Princenton University Press Girardi, L., Bertelli, G., Bressan, A., Chiosi, C., et al. A\& $A$ 391, 195

Mermilliod, J.C.1981, A\&SA 97, 235

Pavani, B.D. \& E. Bica 2007, A\&A (accepted)

Piatti, A.E., Bica, E., Clariá, J.J., Santos Jr., J.F.C., \& Ahumada, A.V. 2002, MNRAS 335, 233

Silva, D.R., \& Cornell, M.E. 1992, ApJS 81, 865 